\title{
Studying penetrating ability of alkaline flow solution of caprolactam production in flint aggregate
}

\author{
Natalia Chumachenko ${ }^{1, *}$, Diana Kizilova ${ }^{1}$, and Ilnur Hafizov ${ }^{1}$ \\ ${ }^{1}$ Samara State Technical University, Academy of Architecture and Civil Engineering, \\ Molodogvardeyskaya St., 194, Samara, 443001, Russia
}

\begin{abstract}
The study focuses on increasing the clinker formation degree of calculus types of siliceous rock. One of the ways to increase the flint aggregate strength is to saturate the surface layer of grains with alkaline ions. While calcining, this will provide formation of confining elements which are more caked than the inner layers. To preserve natural porosity inside granules and to increase density and durability of a surface layer, it is offered to process grains of dense siliceous rock before calcining them with a solution of alkaline flow of caprolactam production (AFCP). It is shown that the degree of density is determined by the soaking depth, which depends on the molecular structure of alkaline solution. The paper also demonstrates results of influence of processing conditions on soaking depth of grains with $10-15 \mathrm{~mm}$ fraction. The researchers studied the effect of AFCP solution concentration in the range varying from 3 to $20 \%$. The duration of treatment ranged from 2 to 5 minutes. The research results are presented in the form of graphs. The optimal processing mode is also determined.
\end{abstract}

\section{Introduction}

The stocks and abundance of siliceous rocks are classified as non-deficient, widely distributed multi-tonnage natural raw materials [1]. Such raw materials are mainly applied in expanded and clinker aggregates production for the construction industry. Herein, the type of the aggregate and the choice of its production technology are determined by the composition and the amount of impurities, as well as, the rock hardness [2-9]. Soft representatives in the form of diatomites and clay diatomites are used to produce expanded aggregates by plastic technology. Calculus types such as gaize, fossil meals are mainly used for sintered aggregates.

All the varieties of siliceous rocks have a natural porosity associated with genesis. Such a structure of raw material favorably affects the properties of the expanded aggregate and is able to play a positive role for the sintered ones.

\footnotetext{
Corresponding author: uvarovang@mail.ru
} 
It is known that the degree of clinker formation of the original natural raw material is determined by the number of meltings. Therefore, for example, lack of meltings in gaizes requires high calcining temperatures when calcining. It is possible to improve clinker formation of the pre-crushed raw material with the help of alkaline additives, but this method irretrievably destroys the natural porosity. Preservation of natural porosity is achieved by crushed stone calcining. Surface treatment of crushed stone with an aqueous solution of alkali metal compounds with the concentration of $0.5-1.0 \%$ during $7-10$ minutes at the first stage, and then with the concentration of 3-5\% during 3-5 minutes, improves clinker formation, strengthens the aggregate, and preserves natural porosity inside the granules [10]. This technology provides the saturation of the surface grain layer with alkaline ions, while calcining provides the formation of a more caked block in comparison with the inner layers. The degree of compaction is determined by the depth of impregnation, which depends on the structure of the alkaline solution molecules.

The crushed stone treatment with an alkaline solution of $\mathrm{NaOH}$ by the known method [10], contributes to the strength increase of the aggregate, but has a number of drawbacks, namely: the aggregate is characterized by low frost resistance and an insufficient degree of sintering. Moreover, the method is expensive due to the use of scarce alkali metal compounds and two-stage treatment.

The strength and frost resistance increase of the siliceous aggregate can be achieved by the method including crushing, surface treatment with an aqueous solution of the sodium salt of adipic acid and cooling. Physical and mechanical characteristics of the aggregate and its durability depend on the thickness of the surface layer saturated with alkaline components, which is determined by the depth of impregnation.

\section{Methods and materials}

To confirm the influence of the surfactant molecules structure on the degree of sintering of the aggregate surface layer, experiments were carried out on the gaize of the Balasheevskoye field in Samara region. Gaize is a dense, light gray rock of sedimentary origin. Chemical composition of the gaize, mass \%: $\mathrm{SiO}_{2}-72.51 ; \mathrm{Al}_{2} \mathrm{O}_{3}-10.05 ; \mathrm{Fe}_{2} \mathrm{O}_{3}-$ 4.78; $\mathrm{CaO}-1.27 ; \mathrm{MgO}=1.13$.

The surface treatment of the siliceous aggregate was carried out with an aqueous solution of an alkaline flow of caprolactam production (AFCP). AFCP [11] is an aqueous solution of sodium salts of acid by-products of air oxidation of cyclohexane. AFCP is a non-flammable brown opaque, liquid without mechanical impurities, its density being 1.1-1.2 $\mathrm{g} / \mathrm{cm}^{3}$ with $\mathrm{pH} 10-13$. The main component of the AFCP is the sodium salts of adipic acid, their mass fraction is $18-30 \%$. Resins up to $10 \%$ and cyclohexane up to $0.8 \%$ can be present as impurities

The gaize was subjected to crushing. After the crushing the $10-15 \mathrm{~mm}$ grains were treated with a solution of AFCP of different concentrations during 2-10 minutes. Solutions of the following concentrations of AFCP: 3, 5, 10, 15 and 20\%. were produced.

\section{Results}

When studying the effect of the concentration of the AFCP and the duration of the treatment on the impregnation depth, 10 samples of the gaize grains were taken for each experiment. The samples were immersed in a solution of the AFCP of the appropriate concentration and treated with stirring for a certain period of time. After the processing, the grain was broken and the impregnation depth was measured. 
Dependences of the effect of the solution concentration and the duration of treatment on the impregnation depth are shown in Figure 1.

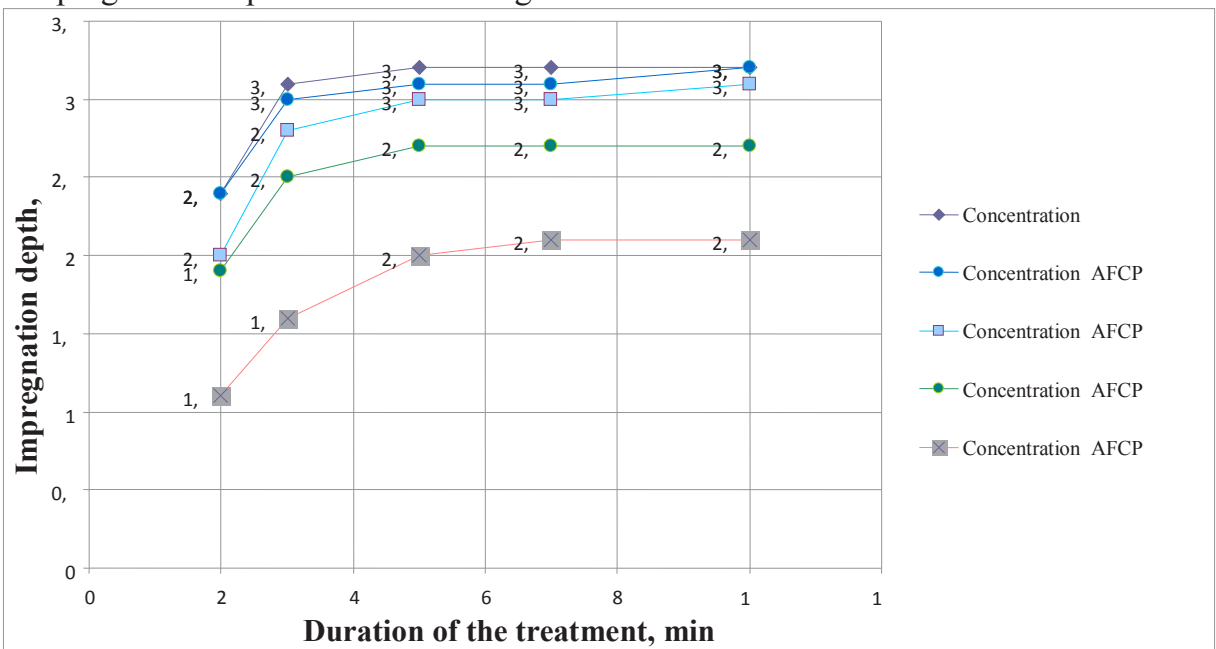

Fig. 1. Influence of the concentration of the AFCP and the processing time on the impregnation depth

As can be seen from figure 1, the concentration of the AFCP solution affects the impregnation depth of siliceous grains. This is especially true when the concentration varies from 3 to $10 \%$. Further increase in concentration has less effect. The depth of impregnation of the grains when treated with a solution of AFCP of 10, 15 and $20 \%$ concentrations has similar values when processing from 2 to 5 minutes, and practically coincide with a longer treatment. When the low-concentration solution (3-5\%) is used, the depth of impregnation increases almost twofold with a change in the treatment time from 2 to 5 minutes. Longer processing practically does not increase the depth of impregnation.

\section{Discussion}

Sodium salt of adipic acid - adipate sodium has the following abbreviated structural formula: $\left(\mathrm{CO}_{2} \mathrm{Na}\right)\left(\mathrm{CH}_{2}\right) 4\left(\mathrm{CO}_{2} \mathrm{Na}\right)$. Adipic acid refers to dibasic carboxylic acids. Carboxylic acids are weak acids, so their salts undergo reversible hydrolysis. The hydrolysis equation is:

$$
\left(\mathrm{CO}_{2} \mathrm{Na}\right) \cdot\left(\mathrm{CH}_{2}\right) \cdot 4\left(\mathrm{CO}_{2} \mathrm{Na}\right)+2 \mathrm{H}_{2} \mathrm{O} \rightarrow \mathrm{HOOC} \cdot\left(\mathrm{CH}_{2}\right) \cdot 4 \mathrm{COOH}+2 \mathrm{NaOH}
$$

Therefore, an alkaline reaction is characteristic for the solution of sodium adipate salts.

Adipic acid molecules consist of polar and nonpolar groups. The polar groups $\mathrm{OH}$ and $\mathrm{COOH}$, and nonpolar - hydrocarbon chains. This duality in the structure determines the behavior of the molecules in the solution as surfactants: the polar group is hydrophilic and the nonpolar hydrophilic, the surfactant molecules are attracted to the phase interface by the hydrophilic part, forming a monomolecular layer.

There are defects in the form of macro- and micro cracks as a result of rock crushing on the surface of grains. When processing such grains with solutions containing a surfactant, the liquid penetrates into the pores and cracks and surface-active substances adsorb on their surface. First of all, surface defects-minute tears or slots of a wedge-shaped section-are subjected to adsorption. Surfactant molecules penetrate into the wedge-shaped microcracks through their slots on the grain surface, cover them with the even (usually monomolecular) adsorption layer and move to its depth along both surfaces to the point where their penetration is limited by the size of the adsorbed molecules. 
Natural siliceous rocks (diatomite, gaize) have a negative charge on the surface. Such a surface adsorbs from the solution the oppositely charged ions. When treated with aqueous solutions of alkaline flow of the production of caprolactam, such ions will be sodium ions.

Positively charged cations are separated from the surface of siliceous rocks by a double layer of water: one layer of firmly bound water around grains of siliceous rocks; another one is a layer of hydrated water around the cations.

In case there are no hydrophobic particles or hydrophobic areas of molecules in the solution, layers of less bound diffusion water are oriented around the cations under the action of their force field after the layer of hydrated water, which promotes the formation of a thickened adsorption layer on the grain surface.

When a dibasic adipic acid is present in the solution, the adipate ion on the surface is planarly fixed on the surface through sodium ions [12]. The hydrophobic hydrocarbon chains of adipate ion eliminate in the adsorption layer a layer of diffusion water (cut off excess water), which, combined with planar fixation on the surface, leads to a sharp decrease in the thickness of the adsorption layer.

The formation of a thin adsorption film has a beneficial effect on the surface layer and on the degree of penetrating power of the solution deep into the grains. In the surface layer, the film intensifies the physicochemical cleavage of the dust-like particles and low-strength areas due to the adsorption strength decrease. Grains treatment with a solution of the alkaline flow of caprolactam production, combined with mixing, provides their removal from the surface.

As the thickness of the adsorption film decreases, the penetrating power of the solution increases, which leads to deeper enrichment of the cracks with sodium ions, and after calcination, the thickness of the caked crust increases and the strength and frost resistance subsequently increased [13].

\section{Conclusion}

1. One way to increase the siliceous aggregate strength is to saturate the surface layer of grains with alkaline ions, which, while calcining, provides the formation of a more agglomerated block in comparison with the inner layers.

2. The degree of compaction is determined by the depth of impregnation, which depends on the structure of the alkaline solution molecules.

3. Research was conducted on the surface treatment of flint aggregate with an aqueous solution of alkaline flow of caprolactam production (AFCP).

4. It has been experimentally established that the thickness of the impregnation layer increases with the increase in the concentration of the AFCP from 3 to $10 \%$, but at a concentration of more than $10 \%$ the changes are insignificant.

5. Optimal treatment parameters were determined: the concentration of the AFCP is 5-10\%; processing time - 3-5 min.

\section{References}

1. M.N. Baranova, S.F. Korenkova, N.G. Chumachenko, Building materials 8, 1-4 (2011)

2. V.N. Ivanenko, Building materials and products from siliceous rocks (Kiev, Budvielnik, 1978)

3. N. G. Chumachenko, D. Galiullina and E. Mironenko, MATEC Web of Conf. 117, 00030 (2017)

4. N.G. Chumachenko, V.V. Tyurnikov, E.V. Petrova, Procedia Engineering 153, 118-123 (2016) 
5. G.A. Kozlov Technology and properties of porous filler based on siliceous rocks for the production of effective wall ceramics, the thesis Abstract on competition of a scientific degree of candidate of technical sciences (Rostov-na-Donu, 2015)

6. GOST R 56507-2015 Fillers thermolithic based on siliceous raw materials. Technical Specification (2015)

7. G.A. Kozlov, Proceedings of the higher education institutions, Building 11-12, 20-24 (2009)

8. V.A. Tyapkin, V.I. Kalashnikov, I.V. Erofeeva, Modern scientific research and innovation 4, Part 1 [Electronic resource]. URL: http://web.snauka.ru/issues/2015/04/51697 (2015)

9. V.D. Kotlyar Wall ceramic products on the basis of siliceous opal-cristobalite rocks gaize, the dissertation Author's abstract on competition of a scientific degree of a Dr.Sci.Tech., Rostov-on-Donu (2012)

10. M.N. Baranova, L.L. Volchek, Method for obtaining a filler, A.c. No. 945136 (USSR), M.C.: From 04 to $31 / 02$, 04/01 B to 31/42. Opubl. 23.07.82. 27, Discoveries. Inventions, 107 (1982)

11. TU 113-03-488-84 AFCP - alkaline flow of the production of caprolactam (production waste of caprolactam) (1984)

12. V.I. Kalashnikov, Bases of plasticization of mineral disperse systems for the production of building materials, the dissertation Author's abstract on the competition of a scientific degree of Doctor of Technical Sciences (Voronezh, 1996)

13. Patent No. 2160722 / N.G. Chumachenko, M.N. Baranova, Y. A. Korenkova Method for obtaining aggregate, IPC 7 C 04B 14/14. 20.12.2000, 35 Discoveries. Inventions. 